\title{
Functionality Evaluation of a Novel Smart Expandable Pedicle Screw to Mitigate Osteoporosis Effect in Bone Fixation: Modeling and Experimentation
}

\author{
Ahmadreza Eshghinejad, ${ }^{1}$ Mohammad Elahinia, ${ }^{2}$ and Vijay K. Goel ${ }^{3}$ \\ ${ }^{1}$ The University of Toledo, Toledo, OH 43606-3390, USA \\ ${ }^{2}$ NiTinol Commercialization Accelerator Dynamic and Smart Systems Laboratory, The University of Toledo, \\ Toledo, OH 43606-3390, USA \\ ${ }^{3}$ Engineering Center for Orthopedic Research Excellence (E-CORE), The University of Toledo, Toledo, OH 43606-3390, USA
}

Correspondence should be addressed to Ahmadreza Eshghinejad; ahmade@uw.edu

Received 9 February 2013; Accepted 7 May 2013

Academic Editor: David Vokoun

Copyright (C) 2013 Ahmadreza Eshghinejad et al. This is an open access article distributed under the Creative Commons Attribution License, which permits unrestricted use, distribution, and reproduction in any medium, provided the original work is properly cited.

\begin{abstract}
This paper proposes a novel expandable-retractable pedicle screw and analyzes its functionality. A specially designed pedicle screw is described which has the ability to expand and retract using nitinol elements. The screw is designed to expand in body temperature and retract by cooling the screw. This expansion-retraction function is verified in an experiment designed in larger scale using a nitinol antagonistic assembly. The results of this experiment are compared to the results of a finite element model developed in Abaqus in combination with a user material subroutine (UMAT). This code has been developed to analyze the nonlinear thermomechanical behavior of shape memory alloy materials. The functionality of the proposed screw is evaluated with simulation and experimentation in a pullout test as well. The pullout force of a normal screw inserted in a normal bone was simulated, and the result is compared with the results of the expandable screw in osteoporotic bone. Lastly, strength of the designed pedicle screw in a foam block is also verified with experiment. The reported finite element simulations and experiments are the proof for the concept of nitinol expandable-retractable elements on a pedicle screw which validate the functionality in a pullout test.
\end{abstract}

\section{Introduction}

Bone screws for various spinal treatments and fixations have been used for about 70 years [1]. Pedicle screws are used as bone anchoring elements to firmly grip the bone to facilitate attachment to the spinal implants. Using the pedicle screws' connection rod, surgeons can fixate the spinal segments together for spinal fusion. The pedicular fixation system (which consists of a minimum of four pedicle screws and the rod) can resist high loads and stabilize a fractured spine. Medical applications of pedicle screws show that tolerating the applied forces is possible for pedicle screws inside a healthy bone. When the bone is not healthy, poor screw purchase becomes the main concern [2].

Osteoporosis is a common bone disease in which the bone mineral density (BMD) reduces. Osteoporosis decreases the bone strength which causes an increased risk of fracture in the bony structures of the patients. This disease is very common in elderly people and steeply increases with age. The main concern of surgeons performing the pedicle screw fixation surgery on patients suffering from osteoporosis is the probability of loosening or pullout failure of the screw during or after surgery [3].

To overcome the drawbacks of osteoporosis in pedicle screw fixations, several methods have been used. Increasing the major diameter of the screw leads to stronger engagement of the screw and bone [4]. Battula et al. [5] showed that the pilot hole size with a diameter larger than $72 \%$ of the screw's outer diameter had adverse effect on the pullout test. Another method is to inject a special kind of biocompatible cement through the screw in order to glue it to the surrounding bone [6]. Even though this method has shown good performance in pullout tests, it poses several disadvantages which restricts its application. One of the main disadvantages is that the 


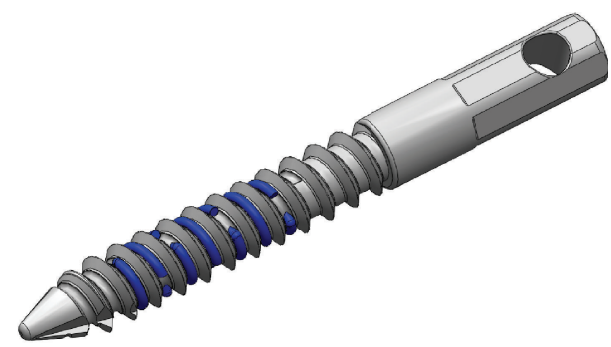

(a)

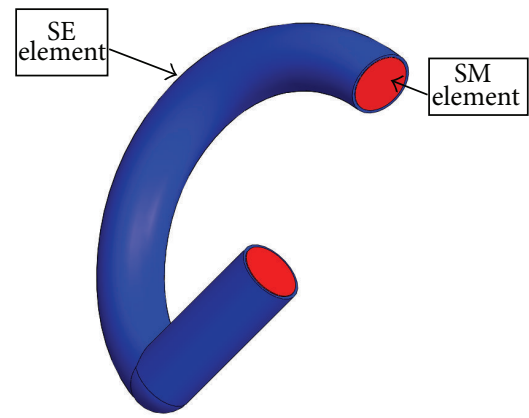

(b)

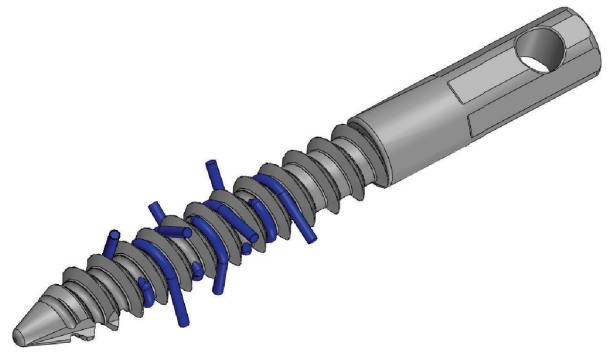

(c)

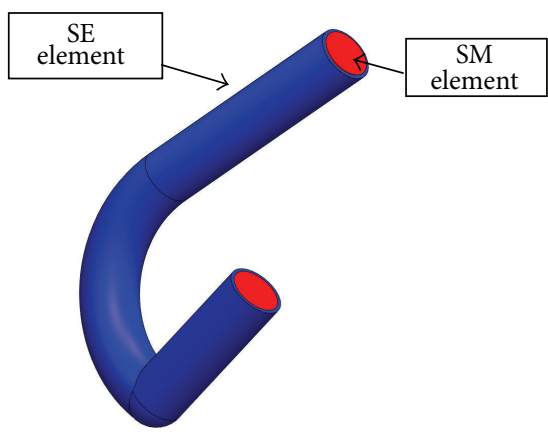

(d)

FIGURE 1: SMART pedicle screw in low temperature in (a). 3D view (b): one retracted nitinol assembly with SM and SE elements and in high temperature form in (b). 3D view (d): one expanded nitinol assembly.

implanted pedicle screw with this method is not removable after insertion.

Some recent researchers have proposed novel methods that take advantage of expandable elements that increase the strength of the connection between the screw and bone $[7,8]$. The elements in these screws are mostly expandable (and not retractable) so that the removal of the screw becomes a much more challenging problem. Later in this paper, we will see the functionality of a proposed expandable-retractable pedicle screw using nitinol, which can solve the problem in more effective way.

\section{Method}

2.1. SMArt Pedicle Screw Design. The proposed pedicle screw takes advantages of the nitinol's superior thermomechanical behavior to serve as the expandable-retractable elements.

2.1.1. Nitinol Thermomechanical Behavior. Nitinol (or NiTi) belongs to a bio-compatible group of alloys known as shape memory alloys. As stated by Lagoudas [9], the special behaviors of this alloy are the results of having two different microstructure states (solid state phases) at low temperature (martensite) and high temperature (austenite). The shape memory effect is the ability of the material to fully recover the introduced strain and reform to the parent phase by heating the material above a certain temperature called austenite temperature. Super-elasticity is characterized by the material's ability to tolerate large strains (up to about $8 \%$ ) without undergoing any plastic deformation. This happens if the temperature is over the austenite final temperature $\left(A_{f}\right)$. In higher temperatures, the material is in austenite phase and has higher modulus of elasticity in specific crystal directions, and at temperatures lower than the material's martensite finish temperature $\left(M_{f}\right)$, it has lower modulus of elasticity in specific crystal directions. This special thermomechanical behavior (along with bio-compatibility, MRI compatibility, and other properties) has been the driving force for recent developments of biomedical devices using nitinol $[10,11]$.

2.1.2. Expandable-Retractable Pedicle Screw Concept. The presented pedicle screw concept consists of a typical pedicle screw and a few additional nitinol assemblies placed around the screw which are designed to have two stable forms. In the low temperature form, the nitinol assemblies retract on the pedicle screw as shown in Figure 1(a). At the body temperature, the second stable form which is the expanded form of the screw is achieved as shown in Figure 1(b). 


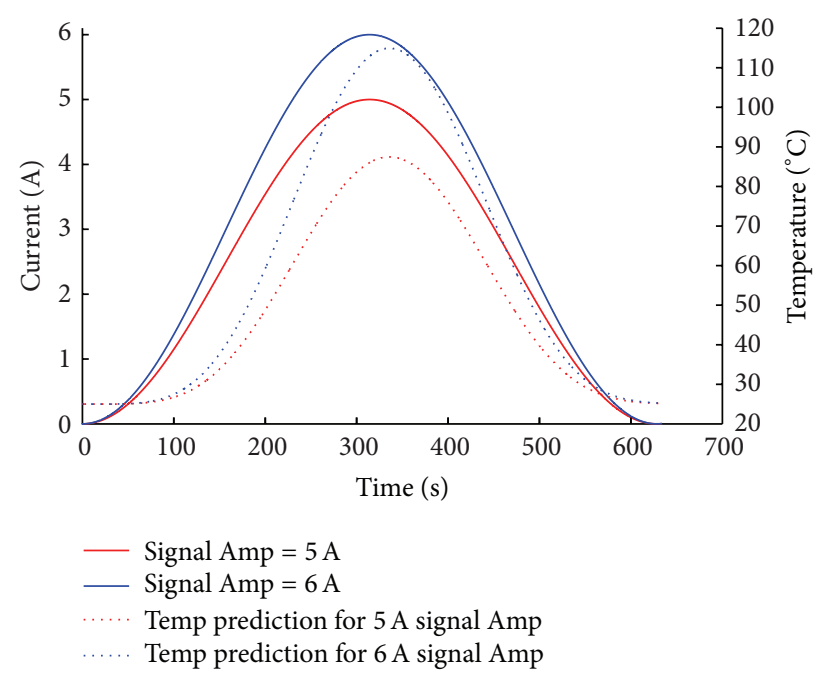

Figure 2: Current input signals to heat the SM wire and the predicted temperature of the SM element due to the signals.

One assembly consists of two nitinol elements. These two elements are assembled in an antagonistic fashion, such that they force each other in the opposite directions. One of them is a super-elastic (SE) element at both low temperature ( $\left.T_{\text {low }}\right)$ outside the body and body temperature $\left(T_{\text {body }}\right)$. This means that the SE material's austenite final temperature $\left(A_{f}^{\mathrm{SE}}\right)$ is lower than both low temperature and body temperature $\left(T_{\text {low }}>A_{f}^{\mathrm{SE}}\right.$ and $\left.T_{\text {body }}>A_{f}^{\mathrm{SE}}\right)$. The other element is a shape memory (SM) material at low temperature which is designed to transform to its austenite phase at body temperature. To this end, the SM material should be selected such that its martensite final temperature $\left(M_{f}^{\mathrm{SM}}\right)$ be higher than the low temperature and its austenite final temperature $\left(A_{f}^{\mathrm{SM}}\right)$ be lower than body temperature $\left(T_{\text {low }}<M_{f}^{\mathrm{SM}}\right.$ and $\left.T_{\text {body }}>A_{f}^{\mathrm{SM}}\right)$. The SE and SM elements original shape should be designed such that at the low temperature, in which the SE element is stronger, the resultant shape be in the retracted form of the assembly (Figure 1(b)). At the body temperature, the SM element passes its austenite start temperature and moves the assembly toward its original shape that is set to be the expanded form (Figure $1(\mathrm{~d})$ ). Now if the surgeon for any reason decides to remove the pedicle screw (e.g., inadequate placement, infection, neural or vascular damage, overcorrection, interference with the facet joints and, etc. [12]) he/she can cool down the screw in order to achieve the retracted form again and unscrew the pedicle screw out of the bone. However, the osteointegration effect is needed to be further investigated with this pedicle screw design. This effect can make the removing impossible after some time.

2.2. Antagonistic SM and SE Expandable Nitinol Elements. To evaluate functionality of the designed expandable-retractable antagonistic nitinol assembly concept, experiments were designed. The finite element (FE) analysis tool in Abaqus software was also validated with the experimental results for future designs.
2.2.1. Experiments. For this experiment, the SE element was a nitinol flat wire with a cross-section of $0.8 \mathrm{~mm} \times 9 \mathrm{~mm}$, and the SM element was a nitinol round wire with a diameter of $2 \mathrm{~mm}$. Both the SM and SE elements were set to have a curved original shape by heating in $500^{\circ} \mathrm{C}$ for 20 minutes and quenching the specimen with water (heat treatment [9]). Note that in the experiment with these sizes, geometries and critical temperatures are used as a proof-of-concept for the expandable-retractable assembly (actuation in two directions). In the final design of this pedicle screw, the antagonistic elements need to be optimized.

The actuator device was made up by assembling the two nitinol elements in an antagonistic fashion (similar to the nitinol assemblies on the designed pedicle screw). To heat up the SM wire, Joule heating was used by applying electric current through the wire. To control the input current to the SM wire, the power supply was controlled by the input signal from a software/hardware control package called dSPACE. To measure the tip displacement of the actuator, a laser distance sensor (optoNCDT 1401) was used (the assembly and the experiment hardware are shown in Section 3). For this experiment, the Joule heating differential equation was simulated to predict the wire temperature (1). Although the resistance of the SMA material slightly changes in the transformation of the states of the material, constant resistance is assumed in this prediction for the sake of simplicity. In this prediction, implemented resistivity $(R)$, specific heat $\left(C_{p}\right)$, density $(\rho)$, and heat convection coefficient $(h)$ are $0.27 \mathrm{Ohm}, 836 \mathrm{j} / \mathrm{Kg} \cdot \mathrm{C}$, $6.5 \mathrm{~g} / \mathrm{cm}^{3}$, and $120 \mathrm{j} /\left(\mathrm{m}^{2} \cdot \mathrm{C} \cdot \mathrm{sec}\right)$, respectively. The predicted temperatures with the Simulink model according to each current input profile are illustrated in Figure 2:

$$
R I^{2}-h_{c} A_{c}\left(T-T_{\mathrm{inf}}\right)=m C_{p} \frac{\mathrm{d} T}{\mathrm{~d} t} .
$$

2.2.2. Finite Element Simulation. In order to better optimize the device, the finite element simulation tool was validated to avoid time expenses of making several prototypes. For this finite element analysis, Abaqus software suite [13] was used. Because of the unique thermomechanical behavior of shape memory alloy materials, a previously developed user-defined material (UMAT) subroutine had to be used in parallel [14]. The UMAT subroutine is the numerical implementation of a full 3D SMA constitutive model [15]. This model is used to predict the behavior of the SM wire which is dependent on the variation in temperature. The SE element is modeled using elastic material properties. The constant superelastic modulus of elasticity was found to be $52 \mathrm{GPa}$. The material properties which were used for simulating the SM wire in the model are shown in Table 1.

Initially, the SE and SM parts were drawn in their shape set form at the low temperature in the software. Next, a rotation displacement was applied to the tip surface of those nitinol elements to make them straight while the assembly was still at the low temperature. In the next step, the required contact between the surfaces of the SE and SM elements was applied. In the same step, SM wire was heated up to reach the highest temperature achieved from the experiment. In the next step, the wire was cooled down to the low temperature 
TABLE 1: Material properties of the SM wire.

\begin{tabular}{lc}
\hline$E_{a}$ & $32 \mathrm{GPa}$ \\
$E_{m}$ & $22 \mathrm{GPa}$ \\
$M_{s}$ & $62^{\circ} \mathrm{C}$ \\
$M_{f}$ & $27^{\circ} \mathrm{C}$ \\
$A_{s}$ & $60^{\circ} \mathrm{C}$ \\
$A_{f}$ & $92^{\circ} \mathrm{C}$ \\
$C_{m}$ & $6 \mathrm{MPa} /{ }^{\circ} \mathrm{C}$ \\
$C_{a}$ & $8 \mathrm{MPa} /{ }^{\circ} \mathrm{C}$ \\
$\varepsilon_{l}$ & 0.02 \\
\hline
\end{tabular}

and again heated up to the high temperature. Since the nitinol wires are used in bending, C3D20R elements were used to avoid the shear locking phenomenon. The contact was chosen to be surface to surface with contact overclosure removal without friction.

2.3. Pullout Test. Pullout test is regarded as a common way of evaluating the mechanical effectiveness of bone screw implants. Performance of the designed pedicle screw was evaluated in a pullout test. The test relies on the fact that the major mode of clinical failure of bone implants is due to normal forces that are pulling the implant out of the bone. During the test, the implanted bone screw resists against a pulling out force. The ultimate pullout force of the screw determines the strength of the fixation. There is an ASTM standard (ASTM F543-02) available to better perform this test.

2.3.1. Finite Element Simulation. In order to simulate the pullout test of the SMArt pedicle screw in the FEA software and evaluate the benefit of the expandable elements, the problem is divided into three sections. First, the pullout test was modeled in Abaqus for a normal screw with healthy bone material properties. The second and third steps were dedicated to the pullout test modeling of the pedicle screw in osteoporotic bone and the effect of the expandable elements.

The pullout test of a normal pedicle screw out of a healthy bone was analyzed by modeling a $6.5 \mathrm{~mm}$ outer diameter bone screw with a thread profile derived from British standards institution [16]. These screw dimensions are used in a similar normal pullout test study by Zhang et al. [17] in Ansys FEA software. The screw is assumed to be made out of stainless steel as reported by Allan and Kenneth [18]. The used modulus of elasticity, Poisson's ratio, and yield stress of the bone were $100 \mathrm{MPa}, 0.2$, and $2.0 \mathrm{MPa}$ (from Goel et al. [19]), and for the screw they were $193,000 \mathrm{MPa}, 0.3$, and 250.0 MPa, respectively.

Because of the symmetry in geometry, one quarter of the screw and the surrounding bone were modeled. This assumption is used by Zhang et al. [17] in their analysis that showed close results to experiments. In this study, the vertebral body was modeled as a block with dimensions of $16 \mathrm{~mm}$ width, $16 \mathrm{~mm}$ height, and $22 \mathrm{~mm}$ length. The position of the pedicle screw was assumed to be in the middle of the block. The threads of the hole in the bone block were assumed to have the same profile as the screw. For this model, a pedicle screw with eight threads measuring $22 \mathrm{~mm}$ in length was used. The outside surfaces of the bone were fixed in all directions. The bone and screw symmetry surfaces were restricted to move only in the direction of the length of the screw. The screw and bone parts were meshed with 4-node linear tetrahedron (C3D4) elements. A finite sliding, surfaceto-surface contact with contact overclosure removal, was used as the contact properties. Hard contact normal behavior was applied to the bone and screw thread surfaces. A friction coefficient of 0.2 was implemented for the contact between surfaces according to Liu et al. [20]. Finally, the top surface of the screw was coupled to the center node of the screw, and a displacement was applied to this node. The graphic of the pullout simulation in this step is depicted in Figure 3.

One of the adverse effects of osteoporosis on the bone mechanical behaviors is the reduction of bone modulus of elasticity as expressed by Dickenson et al. [21]. For the simulation of osteoporotic bone pullout test, the bone modulus of elasticity was assumed to reduce 20 percent due to osteoporosis. Also half of the thread depth in the bone was assumed to be degraded. Element type, boundary conditions, and displacement load were the same as the simulation for the normal bone test.

Advantage of the expandable elements around the screw in pullout test was simulated by assuming full expansion of these elements around the screw. The simulated element was a round wire with $0.4 \mathrm{~mm}$ diameter and $4.5 \mathrm{~mm}$ length. This element was placed in between two of the threads and tangential to the screw surface. The root of the wire is fixed to the screw in all directions. In this study, the effects of other expanded elements were assumed to be the same.

2.3.2. Experiment. The performance of the expanded nitinol elements in pullout test was also verified experimentally. In this experiment, two screws with $1 / 4$ inch thread size, 3.5 inch total length, and 2.75 inch threaded length were used. One of them was the control screw without nitinol expanded elements, and the other one was enhanced with 10 superelastic nitinol elements (austenite final temperature below room temperature). The diameter of the wires was $0.5 \mathrm{~mm}$, and the length was $16 \mathrm{~mm}$. After drilling the required holes on the screw surface between the thread roots, the wires were inserted and fixed into these holes as shown in Figure 4. Since the wires were superelastic, they were expanded around the screw at room temperature. Note that the goal of this experiment was to study the effect of the expanded elements in pullout test. So the SM wires were not added to the assembly, and the SE wires were set in the expanded form on the screw before the experiment.

The two screws were implanted into the drilled holes on two foam blocks by screwing down. The blocks dimensions were $43 \times 43 \times 90 \mathrm{~mm}$ with density of $15 \mathrm{lb} / \mathrm{ft}^{3}$ which is comparable to $5-10 \mathrm{lb} / \mathrm{ft}^{3}$ for osteoporotic bone. The screws were pulled out with the rate of $5 \mathrm{~mm} / \mathrm{min}$ in accordance with ASTM F543-02. Note that this experiment was designed to 


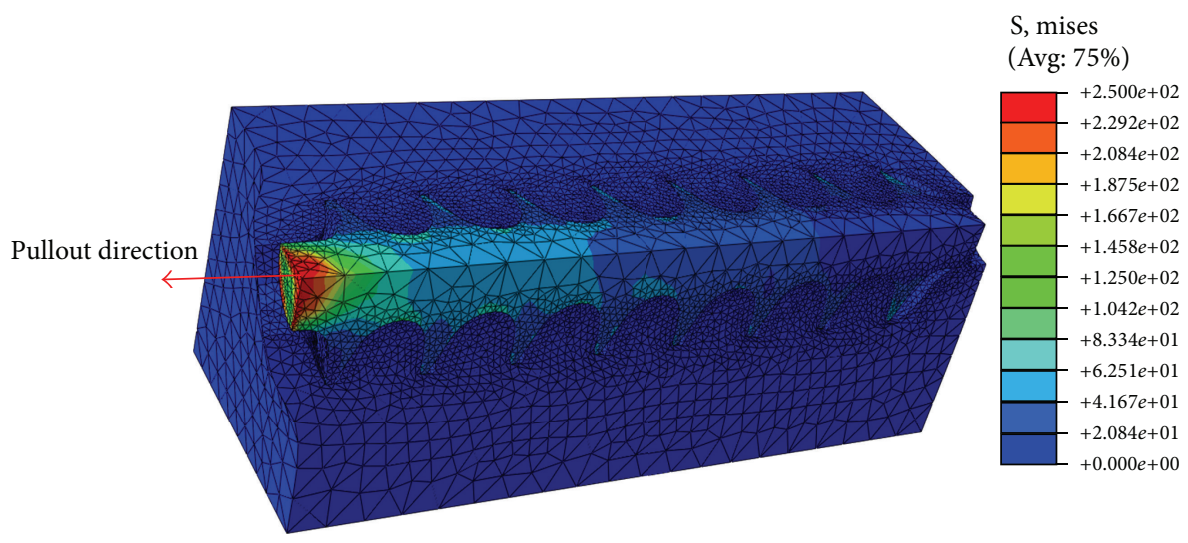

(a)

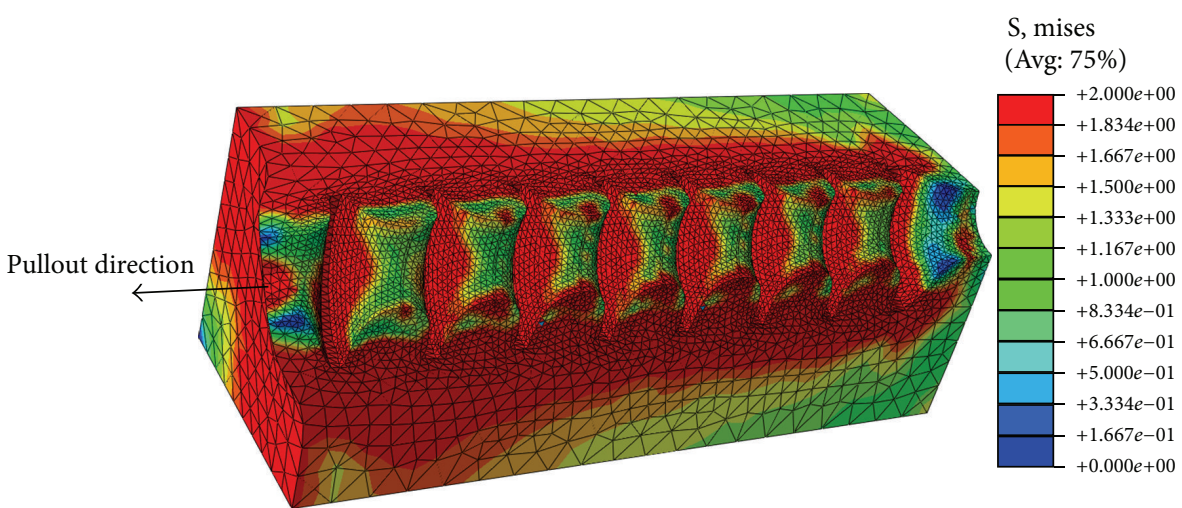

(b)

FIGURE 3: Stress distribution in the deformed quarter of (a) pedicle screw (b) bone (stresses are in MPa).

study only the effect of expandable elements in pullout test and not the expandability and retractably of the elements.

\section{Results}

\subsection{Antagonistic Actuator}

3.1.1. Experimental Results. When the temperature of the assembly was at its low temperature, the SE wire was strong enough to keep the assembly toward the original shape as shown in Figure 5(a). Gradually, by heating the SM wire above its austenite finish temperature, motion at the tip of the actuator was observed. After appropriate heating, no further displacement could be observed by increasing the temperature. By cooling, the achieved displacement decreases to zero, and the room temperature form was achieved. The described heating and cooling process was repeated several times, and a repetitive motion was observed. The observed stable forms at low and high temperatures are desirable for two-way actuation and confirm the expandable-retractable screw concept. Note that the sizes and geometries were used as the proof-of-concept for the design. Also the critical temperatures should be optimized by changing the $\mathrm{Ni}$ ad Ti percentages in the nitinol alloy [9] to match the clinical criteria as explained in Section 2.1.2.
3.2. Finite Element Simulation Results. The predicted shapes of the assembly by finite element, at high and low temperatures, are depicted in Figure 6. These shapes show a striking resemblance to those of the experimental results in Figure 5.

The result of tip displacement against temperature of the assembly is plotted in Figure 7. The maximum displacement of the tip is very well predicted by the simulation. The displacement increases with a steep slope by increasing the temperature beyond the austenite start temperature $\left(60^{\circ} \mathrm{C}\right)$ of the SM element. After adequate heating of the assembly, the actuator reaches its stable form at high temperature. Cooling the wire below its martensite start temperature $\left(62^{\circ} \mathrm{C}\right)$ causes the tip position to move toward its low temperature position. After cooling down to the martensite final temperature $\left(27^{\circ} \mathrm{C}\right)$, the assembly reaches the low temperature stable shape.

\subsection{Pullout Test}

3.3.1. Finite Element Simulation Results. The results of the conducted simulations of the pullout test are illustrated in Figure 8. In the first simulation the pullout force of $550 \mathrm{~N}$ for healthy bone is in agreement with a previous work by Zhang et al. [17]. The simulation results of the next two steps confirm the effect of the expanded elements which can enhance strength of the pedicle screw in an osteoporotic 

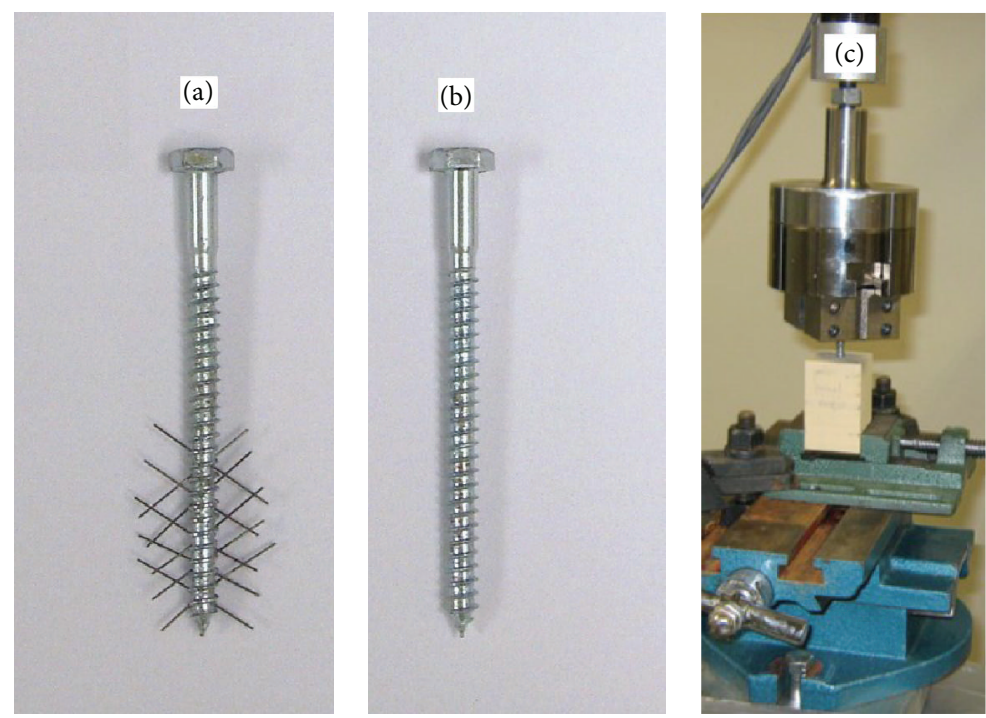

FIGURE 4: (a) SMArt screw enhanced with 10 superelastic wires. (b) Normal screw. (c) Experimental setup. The screw is inserted in the foam block and inserted inside the tensile testing machine.

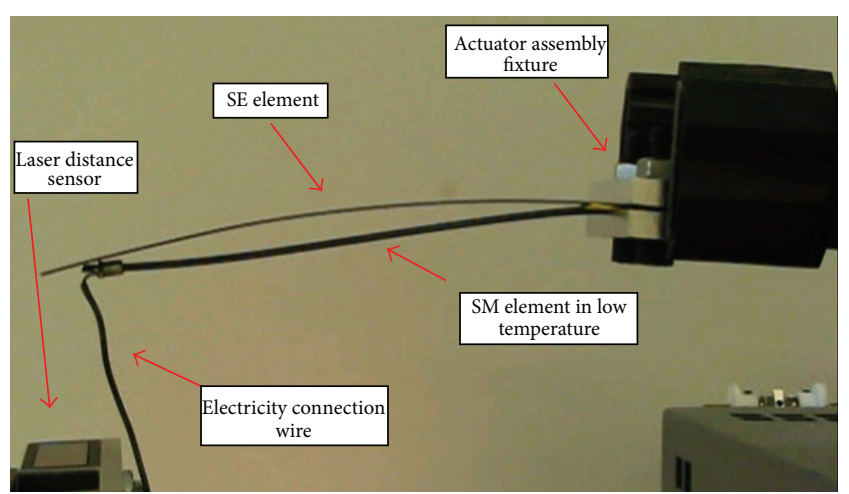

(a)

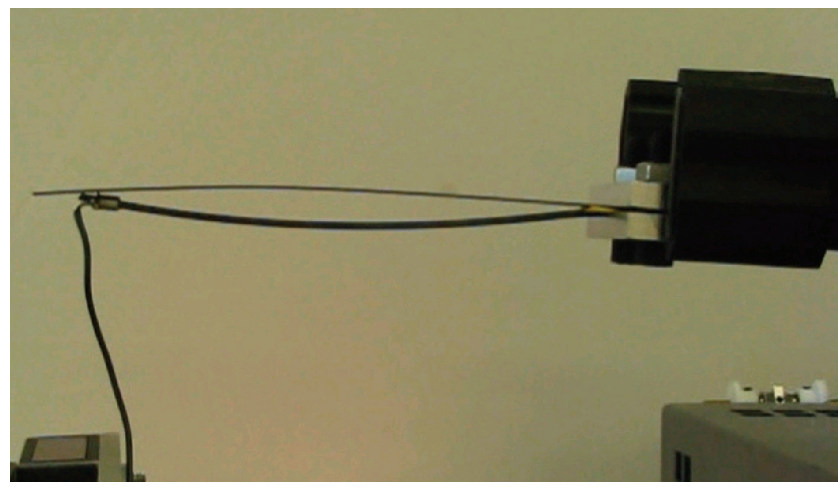

(b)

FIGURE 5: Actuator assembly with the antagonistic SE and SM elements in (a) low temperature (b) and high temperature.

bone. As can be seen by adding six expanded elements to the pedicle screw, the pullout force in a normal bone can be achieved.

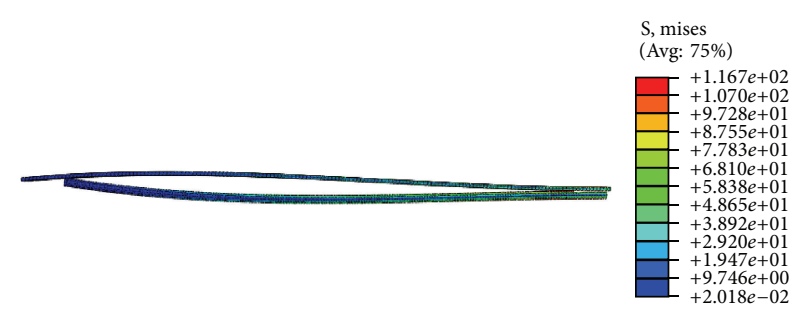

(a)

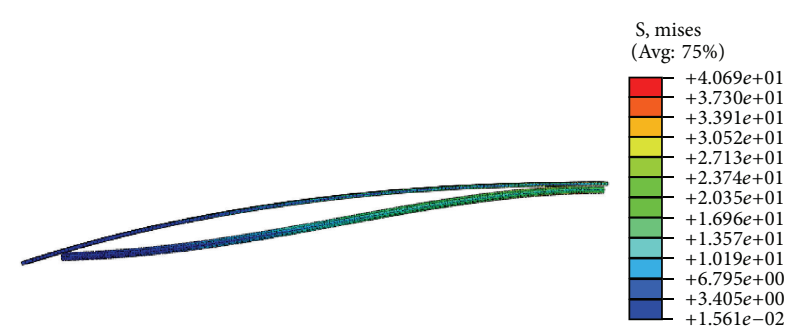

(b)

FIGURE 6: Simulated Mises stress distribution of the SM and SE elements in (a) low temperature and (b) high temperature forms.

3.3.2. Results of Pullout Test Experiment. The results of the explained experiments of pullout test are illustrated in Figure 9. As shown in the plots, the enhanced screw with the expandable elements resists more than the control screw against the pulling out displacement.

\section{Conclusion}

The purpose of the study was to evaluate the functionality and study the concept of a proposed expandable-retractable pedicle screw. The unique part of the design was the assemblies which were placed on the pedicle screw. The assemblies 


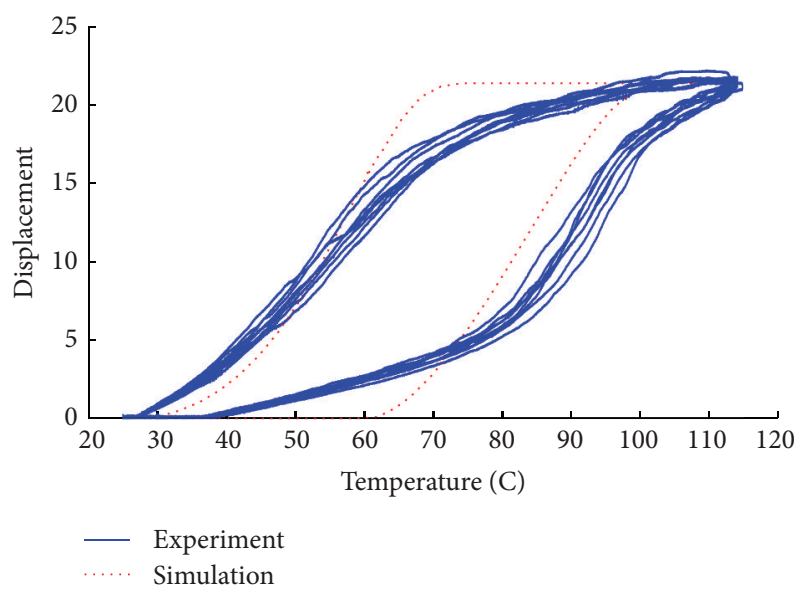

FIgURE 7: Temperature-displacement path of the tip of actuator for the $6 \mathrm{~A}$ current profile as the input.

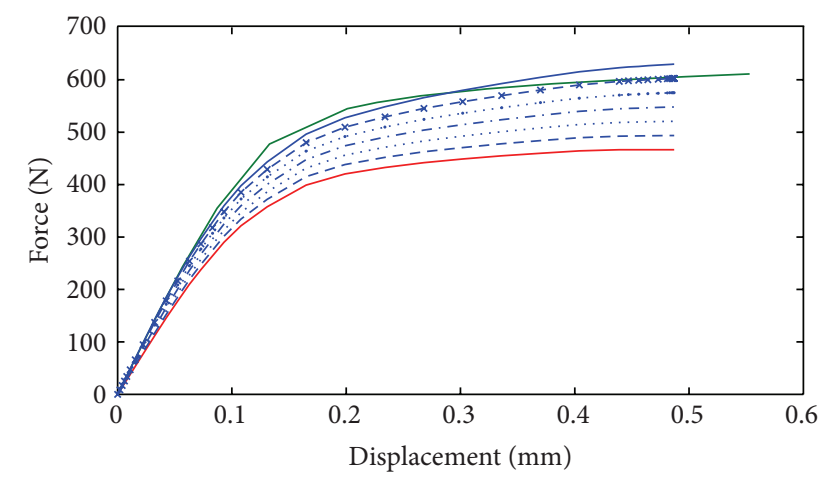

_- Normal bone
- Osteoporotic bone
$\ldots-$ Osteoporotic bone with one expanded wire
$\ldots$. Osteoporotic bone with two expanded wires
$\ldots-$ Osteoporotic bone with three expanded wires
-... Osteoporotic bone with four expanded wires
- Osteoporotic bone with five expanded wires

FIGURE 8: Results of pullout simulations of the pedicle screw in normal bone, osteoporotic bone, and with expandable wires.

were out of nitinol material, which brought the capability of expanding and retracting (at certain temperatures) to the screw.

The functionality of the described actuator assembly was evaluated by making a prototype in larger scale. The reported experiment results showed the desirable results for the concept. The assembly had two different forms at high (body) temperature and room temperature. Obviously, to achieve the final design of the antagonistic nitinol assemblies on the pedicle screw, the material properties for the critical temperatures, shape set forms, cross-section, and length of the wires should be optimized. To be able to optimize these parameters with minimum cost, a reliable simulation software is required. The finite element modeling of the SMA

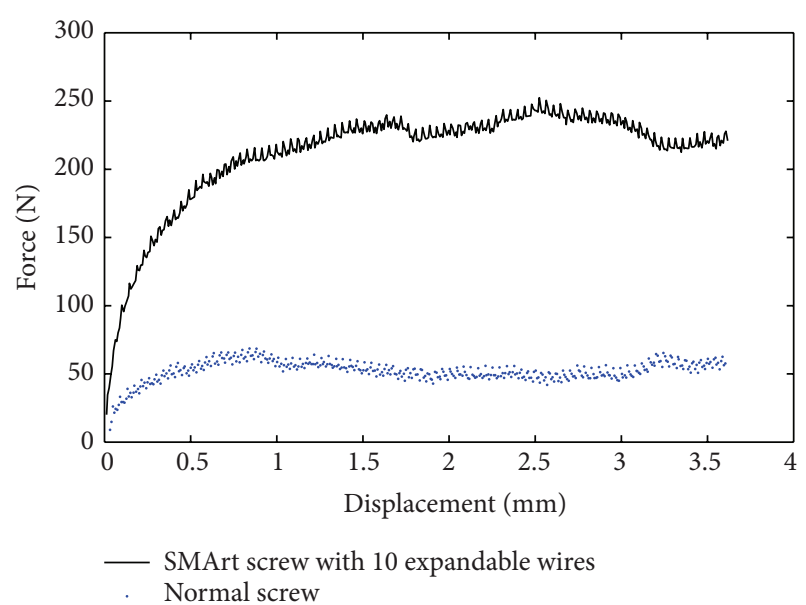

FIGURE 9: Pullout test experimental results with normal screw and with SMArt screw with 10 expandable wires.

material's thermomechanical behavior in Abaqus was verified by comparing the results with the experimental ones.

In both finite element modeling and experiment of the pullout test, the expandable screw showed much higher pullout force than a regular screw. In the finite element simulation, one expanded SMA element fixed on a quarter of a pedicle screw and the surrounding bone using real geometry and bone material properties was simulated. This allowed us to compare the control screw simulation results with previous works and observe the effect of the attached element. While the experiment was conducted in a foam block with regular screw in order to just verify the effect of the expanded elements in experiment. At last, although these results are not comparable, both verified the effectiveness of the proposed pedicle screw in increasing the purchase of the bone screw fixation.

\section{Acknowledgments}

The authors would like to acknowledge the collaboration of Professor Lagoudas, Dr. Darren Hartl, and Mr. Majid Tabesh at Texas A\&M University. Fort Wayne Metals collaborated with the authors in conducting experiments. The authors also would like to acknowledge financial support of Ohio Department of Development through Grant WP 10-010 (Nitinol Commercialization Accelerator).

\section{References}

[1] R. W. Gaines, "The use of pedicle-screw internal fixation for the operative treatment of spinal disorders," Journal of Bone and Joint Surgery. American, vol. 82, no. 10, pp. 1458-1476, 2000.

[2] S. S. Hu, "Internal fixation in the osteoporotic spine," Spine, vol. 22, no. 24, 1997.

[3] S. Inceoglu, Failure of pedicle screw-bone interface: biomechanics of pedicle screw insertion and pullout [Ph.D. thesis], Cleveland State University, 2004.

[4] J. R. Chapman, R. M. Harrington, K. M. Lee, P. A. Anderson, A. F. Tencer, and D. Kowalski, "Factors affecting the pullout 
strength of cancellous bone screws," Journal of Biomechanical Engineering, vol. 118, no. 3, pp. 391-398, 1996.

[5] S. Battula, A. J. Schoenfeld, V. Sahai, G. A. Vrabec, J. Tank, and G. O. Njus, "The effect of pilot hole size on the insertion torque and pullout strength of self-tapping cortical bone screws in osteoporotic bone," Journal of Trauma-Injury Infection \& Critical Care, vol. 64, no. 4, pp. 990-995, 2008.

[6] D. C. Moore, R. S. Maitra, L. A. Farjo, G. P. Graziano, and S. A. Goldstein, "Restoration of pedicle screw fixation with an in situ setting calcium phosphate cement," Spine, vol. 22, no. 15, pp. 1696-1705, 1997.

[7] B. E. McKoy and Y. H. An, "An expandable anchor for fixation in osteoporotic bone," Journal of Orthopaedic Research, vol. 19, no. 4, pp. 545-547, 2001.

[8] M. V. Kotenko, V. A. Kopyssova, V. V. Razdorsky, and V. V. Kishkarev, "Shape-memory dental quadriradical implants for single-stage immediate implantation and undelayed dental prosthetics," Biomedical Engineering, vol. 42, no. 3, pp. 156-158, 2008.

[9] D. C. Lagoudas, Ed., Shape Memory Alloys: Modeling and Engineering Applications, Springer, New York, NY, USA, 2008.

[10] T. Duerig, A. Pelton, and D. Stöckel, "An overview of nitinol medical applications," Materials Science and Engineering A, vol. 273-275, pp. 149-160, 1999.

[11] E. Tarkesh, M. H. Elahinia, M. S. Hefzy, and C. W. Armstrong, "Shape memory alloys, as alternative actuation method for orthosis devices," in North American Congress on Biomechanics, 2008.

[12] J. N. Weinstein, B. L. Rydevik, and W. Rauschning, "Anatomic and technical considerations of pedicle screw fixation," Clinical Orthopaedics and Related Research, no. 284, pp. 34-46, 1992.

[13] Abaqus, Analysis User's Manual, Dassault Systemes of America Corp., Woodlands Hills, Calif, USA, 2009.

[14] M. A. Qidwai and D. C. Lagoudas, "Numerical implementation of a shape memory alloy thermomechanical constitutive model using return mapping algorithms," International Journal for Numerical Methods in Engineering, vol. 47, no. 6, pp. 1123-1168, 2000.

[15] J. G. Boyd and D. C. Lagoudas, "A thermodynamical constitutive model for shape memory materials. Part I. The monolithic shape memory alloy," International Journal of Plasticity, vol. 12, no. 6, pp. 805-842, 1996.

[16] British Standards Institution, Implants for Osteosynthesis. Part 5, Bone Screws and Auxiliary Equipment, (Section 5.3, Specification for the Dimensions of Screws Having Hexagonal Drive Connection, Spherical Under Surfaces and Asymmetrical Thread), British Standards Institution, London, UK, 1991.

[17] Q. H. Zhang, S. H. Tan, and S. M. Chou, "Investigation of fixation screw pull-out strength on human spine," Journal of Biomechanics, vol. 37, no. 4, pp. 479-485, 2004.

[18] T. Allan and J. Kenneth, Biomechanics in Orthopedic Trauma: Bone Fracture and Fixation, M. Dunitz, London, UK, 1994.

[19] V. K. Goel, Y. E. Kim, T.-H. Lim, and J. N. Weinstein, "An analytical investigation of the mechanics of spinal instrumentation," Spine, vol. 13, no. 9, pp. 1003-1011, 1988.

[20] C.-L. Liu, H.-H. Chen, C.-K. Cheng, H.-C. Kao, and W.-H. Lo, "Biomechanical evaluation of a new anterior spinal implant," Clinical Biomechanics, vol. 13, no. 1, pp. S40-S45, 1998.

[21] R. P. Dickenson, W. C. Hutton, and J. R. R. Stott, "The mechanical properties of bone in osteoporosis," Journal of Bone and Joint Surgery. British, vol. 63, no. 2, pp. 233-238, 1981. 

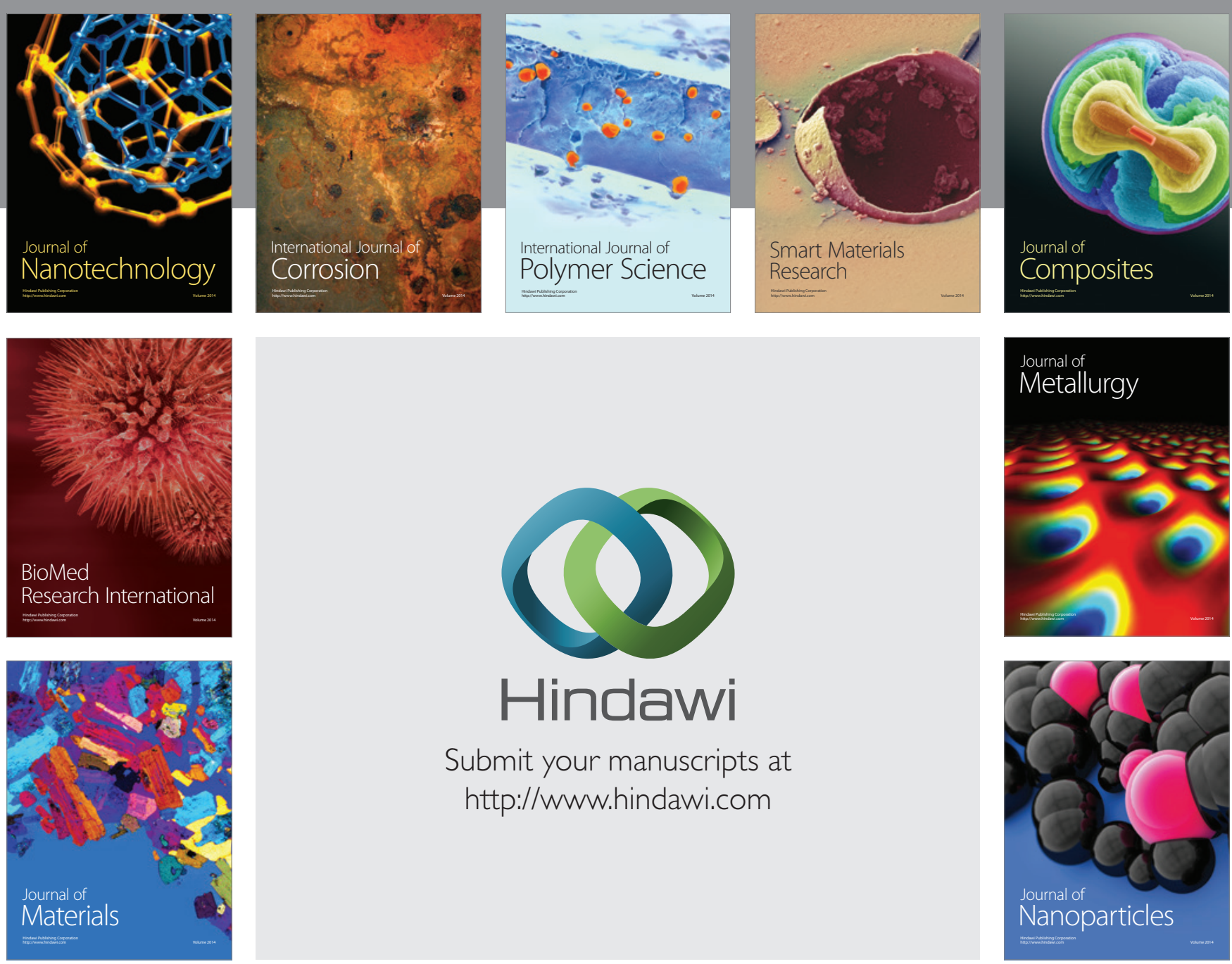

Submit your manuscripts at http://www.hindawi.com
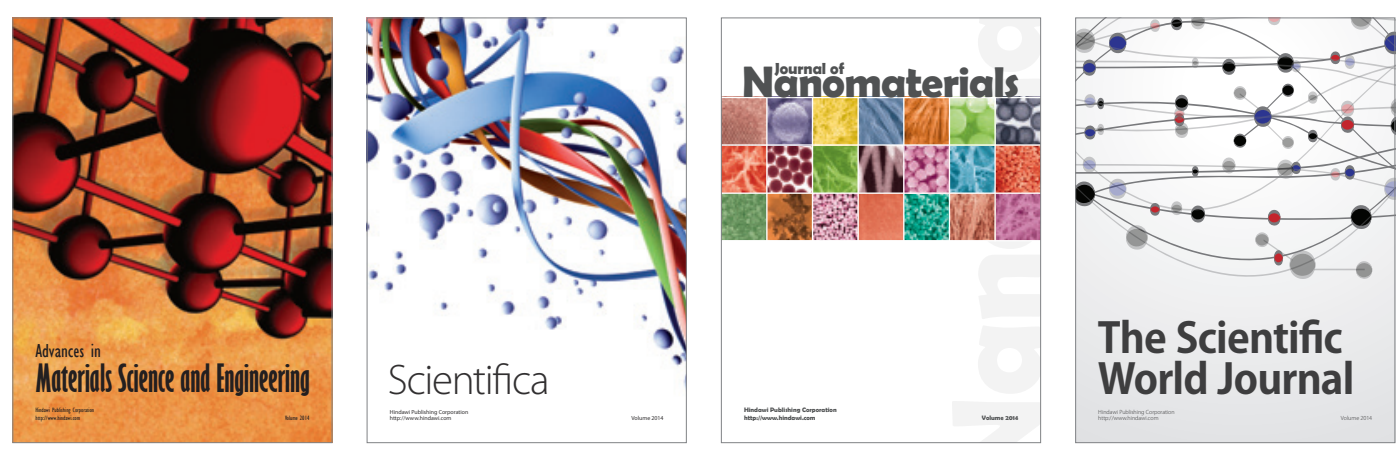

\section{The Scientific World Journal}
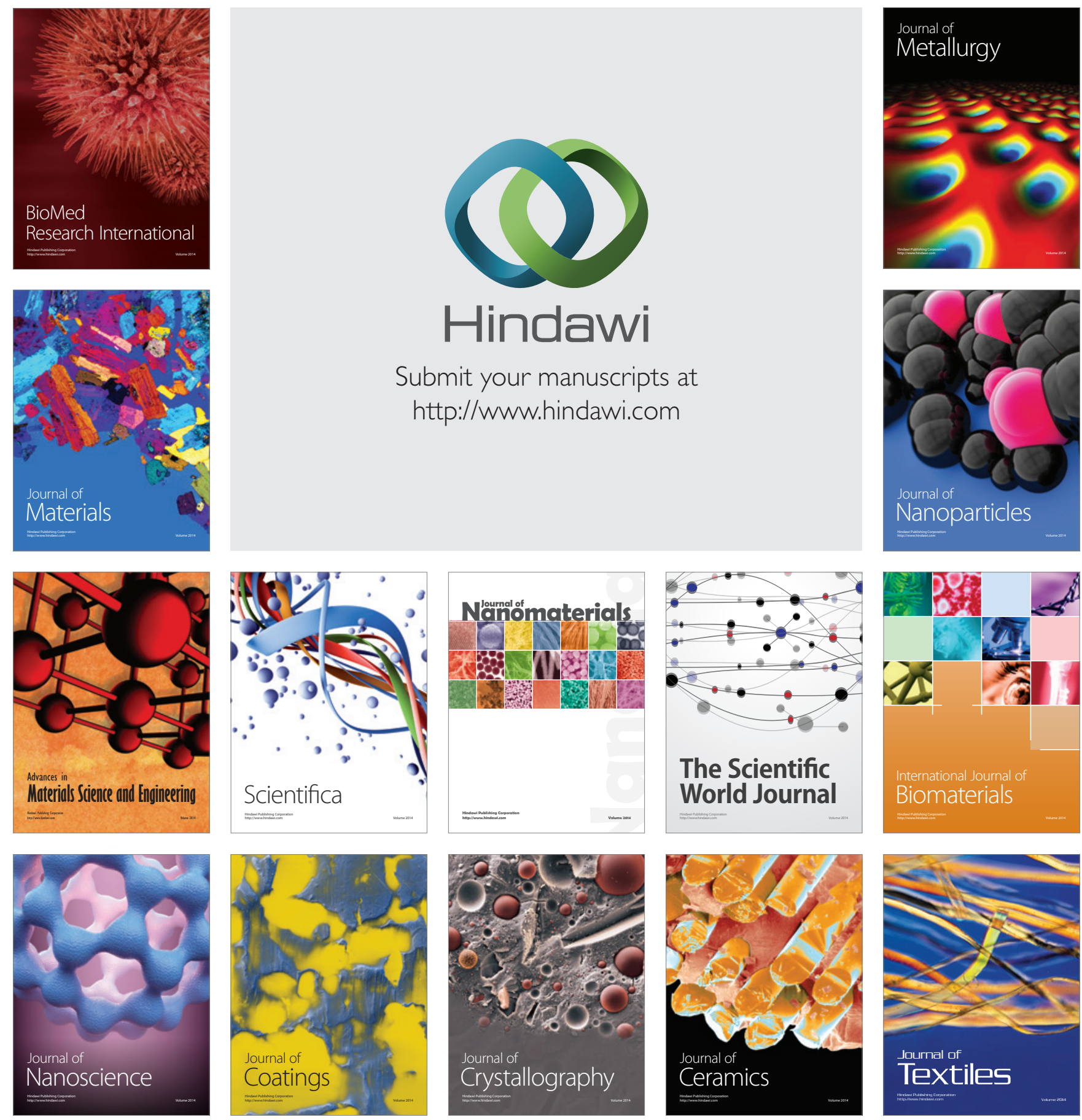